\title{
Pragmatic Recommendations for Tracheostomy, Discharge, and Rehabilitation Measures in Hospitalized Patients Recovering From Severe COVID-19 in Low- and Middle-Income Countries
}

\author{
T. Eoin West, ${ }^{1}$ Marcus J. Schultz, ${ }^{2,3,4 \star}$ Hanan Y. Ahmed, ${ }^{5}$ Gentle S. Shrestha, ${ }^{6}$ and Alfred Papali ${ }^{7}$ for the COVID-LMIC Task Force \\ and the Mahidol-Oxford Research Unit (MORU) \\ ${ }^{1}$ Division of Pulmonary, Critical Care, and Sleep Medicine, University of Washington, Seattle, Washington; ${ }^{2}$ Mahidol-Oxford Tropical Medicine \\ Research Unit (MORU), Mahidol University, Bangkok, Thailand; ${ }^{3}$ Department of Intensive Care, Laboratory of Experimental Intensive Care and \\ Anesthesiology (L.E.I.C.A), Amsterdam University Medical Centers, Location 'AMC', Amsterdam, The Netherlands; ${ }^{4}$ Nuffield Department of \\ Medicine, Oxford University, Oxford, United Kingdom; ${ }^{5}$ Division of Pulmonary and Critical Care Medicine, College of Health Sciences, Addis Ababa \\ University, Addis Ababa, Ethiopia; ${ }^{6}$ Department of Anaesthesiology, Tribhuvan University Teaching Hospital, Kathmandu, Nepal; ${ }^{7}$ Division of \\ Pulmonary and Critical Care Medicine, Atrium Health, Charlotte, North Carolina
}

\begin{abstract}
New studies of COVID-19 are constantly updating best practices in clinical care. However, research mainly originates in resource-rich settings in high-income countries. Often, it is impractical to apply recommendations based on these investigations to resource-constrained settings in low- and middle-income countries (LMICs). We report on a set of pragmatic recommendations for tracheostomy, discharge, and rehabilitation measures in hospitalized patients recovering from severe COVID-19 in LMICs. We recommend that tracheostomy be performed in a negative pressure room or negative pressure operating room, if possible, and otherwise in a single room with a closed door. We recommend using the technique that is most familiar to the institution and that can be conducted most safely. We recommend using fit-tested enhanced personal protection equipment, with the fewest people required, and incorporating strategies to minimize aerosolization of the virus. For recovering patients, we suggest following local, regional, or national hospital discharge guidelines. If these are lacking, we suggest deisolation and hospital discharge using symptom-based criteria, rather than with testing. We likewise suggest taking into consideration the capability of primary caregivers to provide the necessary care to meet the psychological, physical, and neurocognitive needs of the patient.
\end{abstract}

\section{INTRODUCTION}

Recommendations for tracheostomy, hospital discharge, and rehabilitation measures in patients with coronavirus 2019 (COVID-19) have been based primarily on investigations conducted in resource-rich settings from high-income countries (HICs). It may not be practical to apply these recommendations to resource-constrained settings, particularly in low- and middle-income countries (LMICs). Indeed, hospitals and intensive care units (ICUs) in LMICs are frequently restricted in availability of infrastructure, equipment, medications, skilled nurses, and doctors. An international Task Force comprised members from LMICs and HICs, all with direct experience in various LMIC settings, critically appraised a list of questions regarding tracheostomy, hospital discharge, and rehabilitation. We provide a series of recommendations and suggestions on these topics after pragmatic, experience-based appraisal of the literature. A summary of the recommendations is shown in Table 1.

\section{METHODS}

A full description of the methods is provided in the Appendix. In short, we formulated a set of clearly defined questions regarding tracheostomy, hospital discharge, and rehabilitation measures. These questions were reviewed for content and clarity by the entire Task Force. After approval, the group split up, each member seeking evidence for recommendations regarding the questions posed. A literature search was

\footnotetext{
*Address correspondence to Marcus J. Schultz, Department of Intensive Care, Amsterdam University Medical Centers, Location 'AMC', Meibergdreef 9, Amsterdam 1105 AZ, The Netherlands. E-mail: marcus.j.schultz@gmail.com
}

performed in a minimum of one general database (i.e., MEDLINE and EMBASE) and the Cochrane Libraries. Furthermore, we identified investigations from LMICs and searched for unpublished study results.

We selected relevant publications, appraised the evidence, and classified the quality of evidence as high, moderate, low, or very low. Recommendations were rated as strong or weak, depending on the quality of evidence and paying extensive attention to several factors such as availability, feasibility, and safety in LMICs. A strong recommendation was worded as "we recommend ..." and a weak recommendation as "we suggest ..." Recommendations could remain "ungraded" (UG), when, in the opinion of the subgroup members, such recommendations were not conducive for the process described earlier (Appendix Table 2). The recommendations were reviewed by the subgroup in an iterative process and were later reviewed by the entire Task Force in two rounds.

Questions. We formulated two clearly defined questions regarding "tracheostomy," one regarding "hospital discharge" and one regarding "rehabilitation:"

1. In LMICs, where and how should tracheostomies be performed in COVID-19 patients?

2. In LMICs, what preventive and safety measures should be considered during the tracheostomy procedure in COVID19 patients?

3. In LMICs, what are the criteria for hospital discharge of COVID-19 patients?

4. In LMICs, what rehabilitation measures and post-illness sequelae should be considered in COVID-19 patients?

In LMICs, where and how should tracheostomies be performed in COVID-19 patients? Rationale. Patients with COVID-19 may have prolonged respiratory failure requiring mechanical ventilation for many days. Tracheostomy is generally accepted as the most appropriate means to provide this 
TABLE 1

Recommendations and suggestions on tracheostomy and discharge and prolonged care in COVID-19 patients in LMIC (with grading)

1 Tracheostomy

We recommend that tracheostomy on COVID-19 patients be performed in a negative pressure room or negative pressure operating room if possible; if this is not possible, then in a single room with a closed door (strong recommendation and low quality of evidence).

2 Tracheostomy

We recommend that tracheostomy on COVID-19 patients be performed using the technique that is most familiar to the institution and that can be conducted most safely for the patient and the procedural team (UG and best practice statement).

3 Tracheostomy

We recommend that tracheostomy on COVID-19 patients be performed using fit-tested enhanced PPE, with the fewest people required, and incorporating strategies to minimize aerosolization of the virus (strong recommendation and low quality of evidence).

$4 \quad$ Discharge

For patients recovering from severe COVID-19 infection who are clinically appropriate for discharge home, we suggest following local/regional/ national hospital discharge guidelines, where and if available (UG and best practice statement).

6 Deisolation

7 Rehabilitation
5 Discharge

If local/regional/national hospital discharge guidelines are unavailable, we suggest using the following criteria (UG and best practice statement):

1. Afebrile for $\geq 24$ hours AND substantially improved (but not necessarily resolved) respiratory symptoms.

2. No other need to keep the patient hospitalized, such as continued oxygen support. No other compelling need to keep the patient hospitalized.

3. Ability to self-isolate adequately for a minimum of 10 days following the onset of symptoms, if applicable.

If local/regional/national guidelines are unavailable, we suggest deisolation using the following symptom-based criteria for hospitalized patients recovering from severe COVID-19 infection in LMICs (UG and best practice statement):

1. At least 10 days since symptom onset AND

2. At least 24 hours since last fever without the use of antipyretic medications AND

3. Substantial improvement in (but not necessarily resolution of) respiratory symptoms

For patients recovering from severe COVID-19 who are clinically appropriate for discharge home, we suggest taking into consideration the capability of primary caregivers to provide the necessary care to meet the psychological, physical and neurocognitive needs (weak recommendation, low quality of evidence).

LMIC = low- and middle-income countries; PPE = personal protective equipment; RT-PCR = reverse transcriptase $-P C R ; U G$ = ungraded. Grading: See online Supplement Material for explanations. therapy on an ongoing basis. However, there are important factors to weigh regarding the pros and cons of tracheostomy in LMICs, including rehabilitation or long-term care options, cost of care, exposure risk to staff in a resource-constrained environment (as tracheostomy is an aerosol generating procedure and therefore considered high risk for infecting healthcare personnel), and ethical issues related to end-of-life care. The timing of the procedure is discussed in a separate article dedicated to COVID-19 acute respiratory failure management in LMICs (reference-publication pending).

Search results. PubMed was searched through the end of October 2020. The search used combinations of medical subject headings (MeSH) terms and free-text words, including "COVID-19," "coronavirus," "SARS-CoV-2," and "tracheostomy" and "tracheotomy." The search yielded 255 results.

Evidence. No clinical trials were identified, but case reports, case series, and several consensus or guidance documents from a variety of professional organizations and institutions were reviewed. Many of the consensus and guidance documents incorporate data from the SARS outbreak of 2003 to inform recommendations, and others use emerging data from the COVID-19 pandemic. ${ }^{1-12}$ No publication found specifically addressed the approach, feasibility, or safety of tracheostomy in low-income countries. However, multiple publications reported on the successful and safe performance of tracheostomy in COVID-19 patients in both middle- and HICs. ${ }^{13-24}$

As tracheostomy is an aerosol-generating procedure that is associated with the risk of infection to healthcare workers, ${ }^{25}$ in patients with an active SARS-CoV-2 infection, it should ideally be performed in an airborne infection isolation room, whether in an ICU or operating room (OR). ${ }^{26}$ There may be fewer negative pressure rooms available in the OR than in the ICU. Furthermore, in resource-constrained or over-stressed environments, negative pressure rooms may not be available. In such cases, performing the procedure in a single room with a closed and tightly sealed interior door is recommended to minimize flow of contaminated air to other parts of the facility. ${ }^{27}$ For rooms with exterior windows, however, air circulation within the room during the procedure may potentially be increased if the windows are kept open. Natural ventilation is discussed in detail in a separate article in this series dedicated to safety for COVID-19 in LMICs. ${ }^{28}$ Consideration should be given to the need to transport patients to specific procedure rooms as well as to environmental decontamination following the procedure.

Although there is relatively limited literature on tracheostomy for prolonged mechanical ventilation in LMICs, ${ }^{29,30}$ there are two main approaches to performing a tracheostomy in critically ill patients with respiratory failure in HICs: the "open surgical tracheostomy" (OST) and the "percutaneous dilational tracheostomy" (PDT). During the SARS outbreak, there were concerns that PDT resulted in higher risk of viral aerosolization because of the greater airway manipulation required. ${ }^{27}$ Whereas OST is generally performed in the OR requiring relocation of the patient, during the SARS outbreak and in the COVID-19 pandemic, OST has been performed at bedside in the ICU. ${ }^{23,24,27}$ Percutaneous dilational tracheostomy is commonly undertaken at the bedside in the ICU. Open surgical tracheostomy may be performed using electrocautery and suction, which can generate additional aerosols; PDT is frequently performed under direct bronchoscopic visualization, necessitating additional personnel in the room 
and potentially increasing aerosolization of virus from the bronchoscopy procedure. Use of bronchoscopy during PDT also requires that appropriate cleaning protocols for reusable bronchoscopes be in place. Open surgical tracheostomy may be preferred for patients with complex anatomy and is commonly performed by surgeons; PDT may also be performed by pulmonologists and intensivists. No emerging evidence from the COVID-19 epidemic conclusively supports one approach over the other. ${ }^{31-33}$ Given the many considerations, in LMICs, the choice of approach should depend on the patient, the resources available, and local expertise.

Availability, feasibility, affordability, and safety. The availability and feasibility of either OST or PDT in LMICs is likely to depend on the institution. Although PDT may be less expensive because of avoidance of OR charges and anesthetist fees, the cost difference may be reversed if both are performed at the bedside because of the common usage of disposable kits and bronchoscopic guidance for PDT. ${ }^{34}$ Safe performance of both OST and PDT in COVID-19 patients has been reported in adequately resourced settings, ${ }^{13,33}$ but there are fewer safety data on tracheostomy in COVID-19 patients in LMICs. ${ }^{19-21}$

Recommendations and suggestions (Table 1).

1. We recommend that tracheostomy on patients with COVID-19 be performed in a negative pressure room or negative pressure OR if possible; if this is not possible, then in a single room with a closed door (strong recommendation, low quality of evidence).

2. We recommend that tracheostomy on patients with COVID-19 be performed using the technique that is most familiar to the institution and which can be conducted most safely for the patient and the procedural team (UG best practice statement).

In LMICs, what preventive and safety measures should be considered during the tracheostomy procedure in COVID-19 patients? Rationale. Tracheostomy is considered an aerosol generating procedure. When performed in patients with COVID-19, potential aerosolization of SARS-CoV-2 may increase the risk of infection to the proceduralists. Implementation of appropriate preventive and safety measures may reduce these risks.

Search results. PubMed was searched through the end of October 2020. The search used combinations of MeSH terms and free-text words, including "COVID-19," "coronavirus," "SARS-CoV-2," and "tracheostomy" and "tracheotomy." The search yielded 255 results.

Evidence. Tracheostomy was estimated to confer a high risk of infection to healthcare workers during the SARS outbreak. $^{25}$ To protect the proceduralists from exposure to SARS-CoV-2 during tracheostomy, several strategies based on the SARS outbreak are generally recommended. ${ }^{2,4}$ These include carefully planning the procedure in advance; wearing enhanced personal protection equipment (PPE) including purified air-powered respirators (PAPRs) if available, or alternatively N95 respirators together with face shields, minimizing the number of people present in the room, considering neuromuscular blockade to prevent coughing, minimizing electrocautery and suction, maintaining the integrity of the ventilator circuit, and pausing the ventilator when inserting the bronchoscope into the endotracheal tube (for PDT), or after the trachea has been entered and until the tracheostomy tube is securely in place with the cuff inflated (for PDT and OST). It may be prudent to perform an apnea trial before the actual procedure to simulate conditions when ventilation is held during the tracheostomy. ${ }^{35}$

Availability, feasibility, affordability, and safety. The lack of sufficient or appropriately sized PPE (because of availability, cost, or both) for proceduralists in resource-constrained settings, such as in many LMICs, may be a significant limitation to the safe performance of tracheostomy and, therefore, impact feasibility of this procedure. For example, N95 respirators, even if available, may be limited to one size, or may have not been appropriately fit-tested for proceduralists. In addition, conducting the procedure with fewer participants in an isolated environment may impede the ability to respond quickly to any complications that may arise, underscoring the importance of thorough pre-procedural planning and excellent peri-procedural communication among team members.

Recommendations and suggestions (Table 1).

1. We recommend that tracheostomy on patients with COVID-19 be performed using enhanced PPE that includes fit-tested PAPRs or N95 respirators, and face shields or goggles, with the fewest people required, and incorporating strategies to minimize aerosolization of the virus (strong recommendation and low quality of evidence).

In LMICs, what are the criteria for hospital discharge of COVID-19 patients? Rationale. The question of hospital discharge for COVID-19-infected patients is an important one primarily because of concerns for community transmission. Expedited discharge may relieve overburdened hospitals of bed capacity issues, but premature disposition may also heighten the risk of infection spread in the community. Countries with strong public health systems may have sophisticated surveillance networks in place and sufficient human resources available to ensure timely and careful monitoring of patients following discharge. Countries with fragmented public health systems may lack such organization and staffing, leaving discharged patients, their caregivers, and communities at high risk. Furthermore, high population density and overcrowding, particularly in poorer, urban areas of LMICs, may challenge adherence to public health guidelines, such as quarantining and social distancing, and facilitate spread of virus by respiratory droplets. Inadequate sanitation facilities and the resulting impact on hygiene leading to fecal-oral transmission of the virus is also of concern given the high viral loads detected from the gastrointestinal tract. ${ }^{36}$ Thus, timing of hospital discharge for the COVID-19 patient must consider public health safety while balancing facility needs with those of the patient and caregivers.

Search results. PubMed and Google Scholar were searched until May 26, 2020. The search used combinations of MeSH terms and free-text words, including "COVID-19," "coronavirus," "SARS-CoV-2," and "discharge" and "hospitalization." The search revealed 221 citations in PubMed and 1,310 citations in Google Scholar-41 abstracts or links were selected for further review. Bibliographies of available full-text articles were searched for additional references. In addition, a gray literature Google search was performed on May 26 using the search term, "WHO hospital discharge criteria, COVID-19." From this link, the United States and European Centers for Disease Control websites, as well as that of the WHO COVID19 technical guidance page, were accessed. The bulk of the 
relevant literature consisted of retrospective observational studies from China, an upper middle income country. No studies were found from low- or lower middle income countries. Only one randomized clinical trial, also from China, was found that examined rehabilitation-related end points in COVID-19 patients with mild disease. During the review process, a second literature search was performed through December 2, 2020, using the same databases and search terms. The search revealed 1,143 results on PubMed and 364,000 results on Google Scholar. No articles were found specifically in low-income or lower middle income countries. Therefore, a more focused search was performed specifically for the WHO, U. S. CDC, European CDC (ECDC), China CDC (China CDC), and Africa CDC (Africa CDC).

Evidence. "Safe" hospital discharge for patients with severe or critical COVID-19 disease is predicated on clinical factors-that is, the patient's recovery from severe illness-and infectivity-that is, the likelihood of continued viral transmission to others. Although definitive scientific conclusions are elusive at present, the prevailing consensus suggests that prolonged detection of viral RNA via PCR does not necessitate prolonged infectivity. ${ }^{37}$

Early in the pandemic, a small retrospective study from China consisting of 41 patients recovered from severe COVID19 disease suggested a median viral shedding of 31.0 days from illness onset (based on reverse transcriptase-polymerase chain reaction [RT-PCR] testing, not culture of live virus) with no significant age- or gender-related differences. ${ }^{38} \mathrm{An}-$ other very small Chinese retrospective observational study involving four patients recovering from mild to moderate infection demonstrated positive RT-PCR testing following hospitalization, despite twice being tested negative predischarge and despite home isolation and lack of known infectious contacts. ${ }^{39}$ The authors suggest that patients with more severe infection may benefit from longer hospitalization, or closer outpatient observation following discharge, because of the observed prolonged viral shedding.

This suggestion is reinforced by a small German study which examined sputum samples from nine patients in a single cluster, all with mild symptoms due to COVID-19. ${ }^{40}$ Using a combination of viral culture and advanced RT-PCR testing, the authors conclude that there is minimal infection transmission risk for patients past 10 days of symptoms with less than 100,000 viral RNA copies per $\mathrm{mL}$ of sputum. Thus, early discharge to home isolation is reasonable at that inflection point. However, sputum viral loads in the two patients with pneumonia demonstrated later and higher peaks (roughly days 10-11), whereas viral loads were declining at that time for all other patients. A single-center French observational study similarly observed a strong correlation between RT-PCR value and infectivity, suggesting a specific RT-PCR cutoff below which patients are no longer infectious based on correlation with viral culture. Such thresholds are likely to vary between centers and patient populations because of differences in testing kits and testing methods. Thus, the authors suggest that each facility's laboratory develop its own model, by correlation between RT-PCR and viral culture, to augment decision-making regarding hospital discharge and removal of isolation precautions. ${ }^{41} \mathrm{~A}$ retrospective Chinese study involving 94 discharged patients with mild to moderate symptoms demonstrated a significant inverse correlation between PCR-negative conversion and average creatinine kinase
(CK) and lactate dehydrogenase (LDH) levels. The findings suggested a potential benefit to trending biochemical markers of inflammation since LDH and CK levels correlated with viral mRNA elimination. ${ }^{42}$

Many governments specify formal deisolation and discharge criteria for hospitalized COVID-19 patients, but these criteria can vary widely. Whereas some require that patients meet very specific criteria, others offer greater flexibility to facilities and bedside providers. The Chinese National Health Commission's most recent COVID-19 treatment guidelines (September 7, 2020) deem that all of the following criteria must be met for hospital discharge: 1) normal temperature for at least 3 days; 2) significant improvement in respiratory symptoms; 3) demonstrably improved lung imaging; 4) two consecutively negative RT-PCR test results separated by at least 24 hours. Discharged patients are advised to continue home quarantine in an isolated room for 14 days, despite negative testing. ${ }^{43}$ As of the end of May 2020, the national discharge guidelines from Mongolia, a lower middle income country, were nearly identical to those of China (G. Lundeg, personal communication, May 26, 2020). Also as of the end of May 2020 , Kenya, another lower middle income country, recommended hospital discharge when all signs and symptoms of COVID-19 have clinically resolved and patients have two consecutively negative RT-PCR nasopharyngeal swabs taken 24-48 hours apart. Kenya's public health organization did not have specific deisolation criteria at that time but recommended that recovered patients adhere to standard public health hygiene measures applicable to all citizens. (D. Misango, personal communication, May 28, 2020). Haiti, a low-income country, did not specify hospital discharge requirements but requires a single negative RT-PCR test at least 30 days after initial positive testing for the patient to be considered infection free. ${ }^{44}$ Patients are recommended for home isolation until that testing has been completed, although enforcement of isolation is not possible. In May 2020, at the Haitian capital city's only COVID-19 isolation hospital at the time, any patient without fever and not needing oxygen for 4 days was discharged home with verbal instruction on isolation and social distancing. The hospital provided a telephone reminder to patients for repeat PCR testing 3 weeks following discharge (M. Augustin, personal communication, May 25, 2020).

It is important to note that deisolation and discharge criteria tend to evolve along with the science behind COVID-19. For example, on July 17, 2020, the South African National Institute for Communicable Diseases and National Department of Health revised their national deisolation criteria from 14 to 10 days for patients with severe COVID-19 who no longer require oxygen (M. Mer, personal communication, August 3, 2020).

In addition to individual country criteria, several global/ regional health bodies have published guidelines regarding discharge and deisolation for hospitalized patients recovering from COVID-19. At the time of writing, the Africa CDC does not provide formal guidance in this regard. The WHO's third version of COVID-19 clinical management guidelines (published May 27, 2020 ${ }^{45}$ specifies deisolation criteria, but recommends that hospital discharge occur in accordance with local regulations and patient-centered factors, such as need for rehabilitation. For symptomatic patients, irrespective of disease severity, the WHO recommends deisolation after 10 days of symptom onset plus three additional days of no fever or 
respiratory symptoms. For asymptomatic patients, deisolation is recommended 10 days after initial positive testing. If desired, countries may continue to require two negative RTPCR tests spaced at least 24 hours apart as originally recommended by the WHO, but this criterion for testing was removed because of limited availability of and access to laboratories, testing mechanisms and personnel in many LMIC settings.

The U.S. CDC's deisolation and disposition criteria (updated August 10, 2020) vary depending on the severity of illness and the degree to which patients are immunocompromised, but hospital discharge does not require fulfillment of deisolation criteria. In other words, a patient can be discharged even if there is suspicion of continued infectivity. In general, the CDC recommends a symptom-based strategy, unless the patient is severely immunocompromised or persistently symptomatic, in which case a testing-based strategy may be considered. The symptom-based strategy includes the following criteria for patients with severe to critical illness, or for those who have severe underlying immunocompromised states: 1) between 10 and 20 days since onset of symptoms, 2) $\geq 24$ hours since last fever without use of anti-pyretics, and 3) respiratory symptoms have improved. A testing-based strategy, although discouraged in general, should use the same fever and respiratory symptom improvement criteria as for the symptombased strategy but should also include two negative respiratory tract RT-PCR tests spaced at least 24 hours apart. ${ }^{46}$

The original ECDC hospital discharge guidelines from April 2020 varied depending on the local epidemiological situation. ${ }^{47}$ For low disease-burden situations and with optimal testing capacity, patients were recommended for discharge based on clinical criteria (no fever for $>3$ days, improved respiratory symptoms, improved pulmonary imaging, no other need for further hospitalization, and clinician assessment) and laboratory evidence of virus clearance in respiratory samples (at least two negative RT-PCR tests from nasopharynx or throat spaced apart $\geq 24$ hours and a minimum of 7 days after the first positive RT-PCR test). In high local disease burden or strained testing capacity situations, discharge was recommended, when clinically appropriate, with $\geq 14$ days selfisolation, provided that these patients receive regular public health monitoring and have suitable facilities in which to isolate. The ECDC updated guidelines, published October 16, 2020 , similarly to those of the U. S. CDC, use a combination of symptom-based and testing-based strategies, depending on the severity of illness. ${ }^{48}$ These European discharge and deisolation guidelines are summarized in Figure 1.

One common denominator between these various hospital discharge guidelines is outpatient follow-up. In LMICs, inperson or video/telemedicine follow-up may be sporadic or not feasible for different reasons; however, the large penetration of cellular phones in LMIC environments suggests that telephone follow-up may be possible. A pilot telemedicine program has been developed in Toronto, Canada, to follow symptoms and to communicate with family members of COVID-19 patients. ${ }^{49}$ Approximately one-third of patients enrolled in this program were followed telephonically. Similar programs could be considered for LMICs, although the burden may fall on individual health facilities in the absence of governmental coordination.

Availability, feasibility, affordability, and safety. Numerous factors germane to the resource-limited settings in LMICs can render hospital disposition difficult. Many guidelines mandate RTPCR testing for discharge and for removal of isolation precautions. Such testing may not be widely available and thus may delay discharge. Furthermore, who pays for testing and how much such testing costs may also come into play. The actual cost of RT-PCR testing varies widely between and even within LMICs. ${ }^{50}$ While no reports could be found of patients or families being responsible for the costs of testing, it is plausible that, in such a scenario, a patient could refuse testing because of its expense. The absence of strong public health surveillance mechanisms could also mean that patients might be lost to follow-up after discharge, thereby increasing risk of community transmission. Even for those patients who desire follow-up testing, difficulty accessing such testing due to lack of testing locations, lack of transportation, lack of financial means, etc., may preclude this possibility.

Recommendations and suggestions (Table 1).

1. For hospitalized patients recovering from severe COVID-19 infection in LMICs, we recommend following local/ regional/national deisolation and hospital discharge guidelines, where available (UG and best practice statement).

2. If local/regional/national guidelines are unavailable, we suggest hospital discharge using the following symptom-based criteria for hospitalized patients recovering from severe COVID-19 infection in LMICs (UG and best practice statement):

I. Afebrile for $\geq 24$ hours AND substantially improved (but not necessarily resolved) respiratory symptoms.

II. No other need to keep the patient hospitalized, such as continued oxygen support.

III. Ability to self-isolate adequately for a minimum of 10 days following the onset of symptoms, if applicable.

3. If local/regional/national guidelines are unavailable, we suggest deisolation using the following symptom-based criteria for hospitalized patients recovering from severe COVID-19 infection in LMICs (UG and best practice statement):

I. At least 10 days since symptom onset AND

II. At least 24 hours since last fever without the use of antipyretic medications AND

III. Substantial improvement in (but not necessarily resolution of) respiratory symptoms

4. Upon hospital discharge, we recommend that all patients and caregivers receive comprehensive education on adequate hygiene and the importance of mask-wearing, including for close contacts (UG and best practice statement).

In LMICs, what rehabilitation measures and post-illness sequelae should be considered in COVID-19 patients? Rationale. In addition to infection and epidemiological concerns that impact when to discharge, the question of how and where to discharge hospitalized patients who have recovered from COVID-19 infection is essential. Prolonged post-discharge symptoms among COVID-19 survivors are well described, ${ }^{51}$ but critical illness myopathy, polyneuropathy, and post-intensive care syndrome (PICS) are particularly relevant. ${ }^{52,53}$ In resourcerich settings, discharge to dedicated rehabilitation facilities or targeted outpatient management often is recommended to address these long-lasting neurocognitive and musculoskeletal sequelae of critical illness. In resource-restricted settings in LMICs, such services may be less available, or less readily accessible. Patients discharged home without dedicated follow-up; therefore, may have poorer long-term outcomes. ${ }^{54}$ Thus, the timing of hospital discharge in LMICs must weigh the short- and 


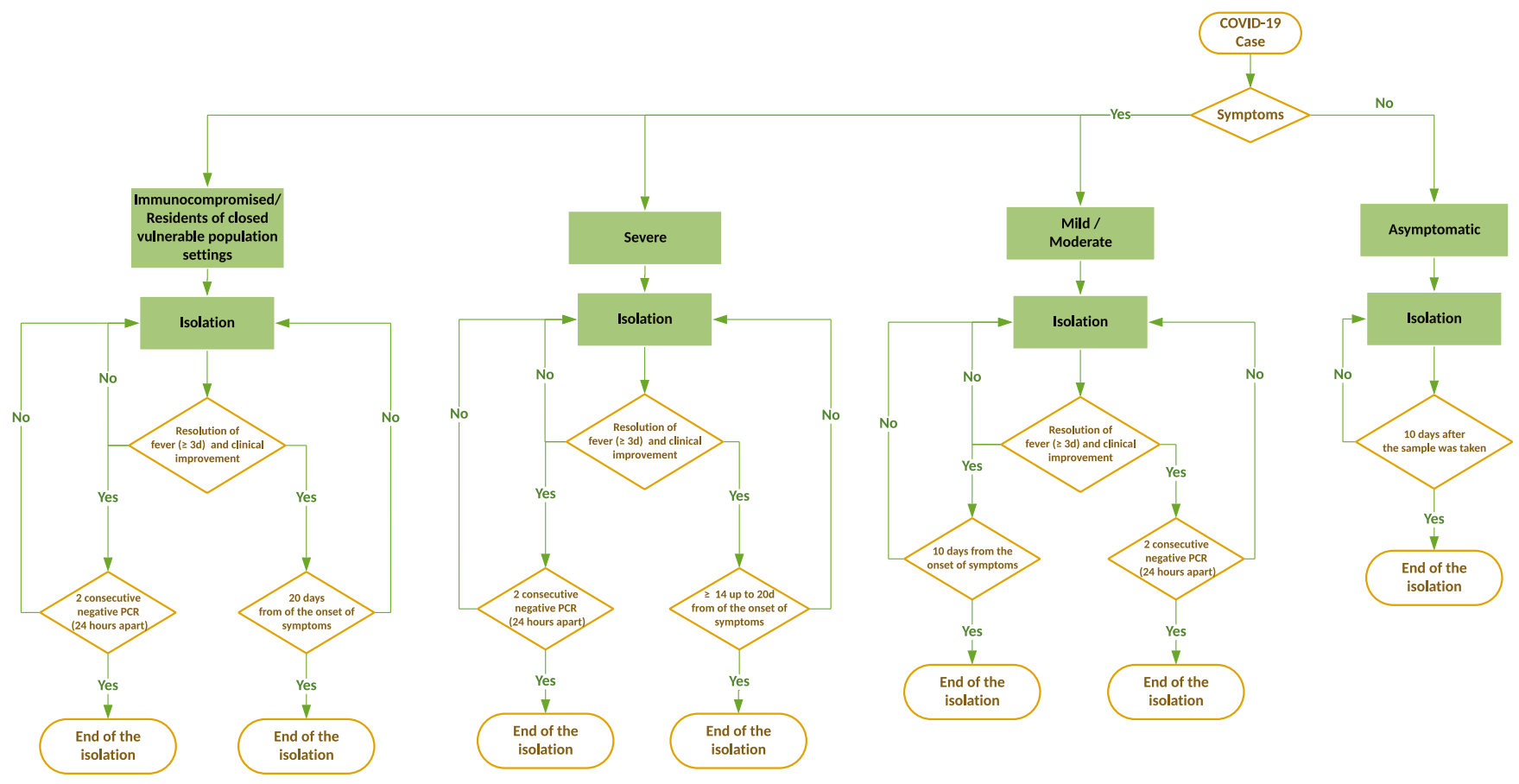

FIGURE 1. ECDC COVID-19 deisolation criteria (adapted from the European CDC. Technical Report. Guidance for discharge and ending of isolation of people with COVID-19. Available at: https://www.ecdc.europa.eu/sites/default/files/documents/Guidance-for-discharge-and-endingof-isolation-of-people-with-COVID-19.pdf. Accessed December 3, 2020). ECDC = European CDC. Reprinted with permission of the European Centre for Disease Prevention and Control.

medium-term needs of the convalescing COVID-19 patient experiencing the sequelae of critical illness and the caregivers' capacity to provide adequate post-discharge care against the facility's imperative to optimize bed capacity.

The very high burden of chronic musculoskeletal and neurocognitive deficits among critical illness survivors ${ }^{55}$ can be addressed by early mobilization in the hospital and multidisciplinary post-discharge rehabilitation. The role of rehabilitation specialists and prevalence of PICS for critical illness survivors in LMICs is ill-defined, and even less so for COVID-19. Although the WHO's Rehabilitation "2030: A Call for Action," created in response to the 2003 SARS epidemic may provide contemporary guidance, ${ }^{56}$ only select LMICs have published data on ICU physiotherapy. ${ }^{57}$

Search results. PubMed and Google Scholar were searched on May 25, 2020. The search used combinations of MeSH terms and free-text words, including "COVID-19," "coronavirus," "SARS-CoV-2," "discharge," "PICS," and "intensive care syndrome." Search results revealed only one citation in PubMed and 3,090 citations in Google Scholar. Bibliographies of available and relevant full-text articles were searched for additional references. One randomized clinical trial from China involving COVID-19 patients, was found, along with several commentaries. During the review process, another search was performed on December 3, 2020. A prospective Russian study and updated consensus rehabilitation guidelines from China and European/American professional societies were added.

Evidence. A statistically limited, prospective, single-ICU study from Russia suggested that inpatient pulmonary rehabilitation using a novel rehabilitation protocol improved oxygen demand in non-ventilated patients. ${ }^{58}$ Specific to LMICs, only one, single-center randomized clinical trial exists (from China, an upper middle income country) comparing 6 weeks of post-discharge respiratory rehab versus none for elderly (age $>65$ years) COVID19 survivors. The severity of illness of enrolled patients was not disclosed, and there was neither mention of critical illness or ICUlevel care for any patient, nor discussion of healthcare worker protection with personal protective equipment. The investigators found that patients who participated in the rehabilitation program witnessed significant improvements in pulmonary function testing parameters, 6-minute walk test distance, patient-reported anxiety, and quality of life, but did not experience significant reduction in self-reported depression. ${ }^{59}$ An ongoing randomized clinical trial of inpatient tele-rehabilitation is currently recruiting in a Wuhan, China COVID-19 hospital. ${ }^{60}$ The results of this study will help to inform revisions of the proposed Chinese recommendations for pulmonary rehabilitation of adult COVID-19 patients. ${ }^{61}$ These recommendations are based primarily on expert opinion and include the following parameters: 1) for nonsevere inpatients, pulmonary rehabilitation is recommended to relieve the symptoms of dyspnea, anxiety, and depression, physical function, and quality of life; 2) for severe/critical inpatients, the early performance of pulmonary rehabilitation is not suggested; 3) for isolating patients, the pulmonary rehabilitation guidance should be conducted through education video, instruction manual, or remote consultation; 4) assessment and monitoring should be performed throughout the entire pulmonary rehabilitation process. Separately, a joint Chinese professional society guideline for outpatient pulmonary rehabilitation for severe COVID-19 survivors also has been published. ${ }^{62}$ Unlike the Chinese inpatient guidelines, consensus rehabilitation guidelines published by the European Respiratory Society and American Thoracic Society strongly advocate for rehabilitation at/around the bedside, both in the ICU and on the medical ward, until discharge. ${ }^{63}$ 
Post-intensive care syndrome represents an increasingly recognized group of long-term physical, psychiatric, and neurologic complications related to the critical illness period. ${ }^{64}$ The most robust PICS literature stems from the acute respiratory distress syndrome (ARDS) adult patient population in HICs. Although PICS has not yet been well described in the COVID-19 population, the frequent observation of ARDS in COVID-19 patients portends an equally high anticipated incidence of PICS among COVID-19 survivors.

There is scant PICS literature available from LMICs. A 2018 systematic review and meta-analysis revealed four randomized clinical studies and one uncontrolled study, all performed in HICs. Investigators were unable to perform secondary analysis of differences between high- and low-income countries due to lack of studies from LMICs. ${ }^{65}$ Another meta-analysis revealed substantial rates of joblessness and delayed return to work among ICU survivors, ${ }^{66}$ which can have negative downstream effects on individual and national economic productivity. In LMICs, where the hospitalized patient with severe COVID-19 respiratory infection might represent a family's sole breadwinner or caregiver, the immediate economic impact could be catastrophic.

Availability, feasibility, affordability, and safety. Lack of recognition among LMIC-based providers of delirium and critical illness myopathy/polyneuropathy (also known as ICU-acquired weakness) may contribute to nonadherence to the best practices of early mobilization for hospitalized patients. Moreover, limited human resources and discharge options for hospitalized patients in many LMICs may preclude the ability for survivors of critical illness, whether COVID-19 or otherwise, to receive dedicated care for the long-term sequelae. ${ }^{63}$ The burden of post-hospitalization care will fall squarely on the shoulders of family members, who may be unfamiliar with and ill equipped to face the medium- to long-term musculoskeletal and neurocognitive deficits that their loved ones may endure. Further research is required in highincome country (HIC) and LMIC settings alike to understand how to optimize care for critical illness survivors. The nuances and socioeconomic challenges characteristic of many resourcelimited settings in LMICs, however, warrants dedicated study.

Recommendations and suggestions (Table 1).

1. For patients recovering from severe COVID-19 who are clinically appropriate for discharge home, we suggest taking into consideration the capability of primary caregivers to provide the necessary care to meet the psychological, physical, and neurocognitive needs (weak recommendation and low quality of evide"nce).

Received September 10, 2020. Accepted for publication January 4, 2021.

Published online January 13, 2021.

Note: The Appendix can be found at www.ajtmh.org.

Financial support: Open access fees for this article and all others in this issue were supported by the Wellcome Trust of Great Britain.

Authors' addresses: T. Eoin West, Division of Pulmonary, Critical Care, and Sleep Medicine, University of Washington, Seattle, WA, E-mail: tewest@uw.edu. Marcus J. Schultz, Mahidol-Oxford Tropical Medicine Research Unit (MORU), Mahidol University, Bangkok, Thailand, Department of Intensive Care, Laboratory of Experimental Intensive Care and Anesthesiology (L.E.I.C.A), Amsterdam University Medical Centers, Location 'AMC', Amsterdam, The Netherlands, and Nuffield Department of Medicine, Oxford University, Oxford, United Kingdom, E-mail: marcus.j.schultz@gmail.com. Hanan Y. Ahmed, Division of Pulmonary and Critical Care Medicine, College of Health Sciences, Addis Ababa University, Addis Ababa, Ethiopia, E-mail: hananyusufahmed@gmail.com. Gentle S. Shrestha, Department of Anaesthesiology, Tribhuvan University Teaching Hospital, Kathmandu, Nepal, E-mail: gentlesunder@hotmail.com. Alfred Papali, Division of Pulmonary and Critical Care Medicine, Atrium Health, Charlotte, NC, E-mail: alfred.papali@atriumhealth.org.

This is an open-access article distributed under the terms of the Creative Commons Attribution (CC-BY) License, which permits unrestricted use, distribution, and reproduction in any medium, provided the original author and source are credited.

\section{GROUP MEMBERS OF THE "COVID-LMIC TASK FORCE"}

Heads: Alfred Papali (Atrium Health, Charlotte, NC) and Marcus Schultz (Mahidol University, Bangkok, Thailand; University of Oxford, Oxford, United Kingdom; Amsterdam University Medical Centers, location 'AMC', Amsterdam, The Netherlands).

Advisors: Neill K. J. Adhikari (Interdepartmental Division of Critical Care Medicine, University of Toronto, Toronto, Canada); Emilie J. Calvello Hynes (University of Colorado School of Medicine, Department of Emergency Medicine, Denver); Martin Dünser (Kepler University Hospital and Johannes Kepler University Linz, Linz, Austria) and Mervyn Mer (University of the Witwatersrand, Johannesburg, South Africa).

Subgroup members, in alphabetical order: Andrew Achilleos (Sunnybrook Health Sciences Centre, Toronto, Canada); Hanan Y. Ahmed (Division of Pulmonary and Critical Care Medicine, College of Health Sciences, Addis Ababa University, Addis Ababa, Ethiopia); Kevan M. Akrami (Universidade Federal da Bahia, Salvador, Brazil; University of California San Diego, San Diego); Lia M. Barros (University of Washington, Seattle, WA); B. Jason Brotherton (AIC Kijabe Hospital, Kijabe, Kenya); Sopheakmoniroth Chea (Calmette Hospital, Phnom Penh, Cambodia); William Checkley (The Johns Hopkins University School of Medicine and The Johns Hopkins University Center for Global Health, Baltimore, MD); Elif Cizmeci (Interdepartmental Division of Critical Care Medicine, Sunnybrook Health Sciences Centre, University of Toronto, Toronto, Canada); Natalie Cobb (University of Washington, Seattle, WA); Finot Debebe (Department of Emergency Medicine and Critical Care, Addis Ababa University School of Medicine, Addis Ababa, Ethiopia); Juliana Ferreira (University of São Paulo, São Paulo, Brasil); Gabriela Galli (University of Pittsburgh School of Medicine, Pittsburgh, PA); Tewodros Haile (Department of Internal Medicine, College of Health Sciences, Addis Ababa University); Bhakti Hansoti (Department of Emergency Medicine, Johns Hopkins University School of Medicine, Baltimore, MD); Madiha Hashmi (Ziauddin University, Karachi, Pakistan); Rebecca Inglis (Lao-Oxford-Mahosot Hospital-Wellcome Trust Research Unit (LOMWRU), Mahosot Hospital, Vientiane, Lao People's Democratic Republic and Department of Intensive Care, Oxford University Hospital Trust, Oxford, United Kingdom); Burton W. Lee (National Institutes of Health, Bethesda, USA; University of Pittsburgh School of Medicine, Pittsburgh, PA); Faith Lelei (AIC Kijabe Hospital, Kijabe, Kenya); Ganbold Lundeg (Mongolian National University of Medical Sciences, Ulan Bator, Mongolia); David Misango (The Aga Khan University, Nairobi, Kenya); Ary Serpa Neto (Department of Critical Care Medicine, Institute of Education and Research, Hospital Israelita Albert Einstein, São Paulo, Brazil; Department of Intensive Care, Amsterdam University Medical Centers, location "AMC," University of Amsterdam, Amsterdam, The Netherlands); Alfred Papali (Atrium Health, Charlotte, NC); Casey Park (Interdepartmental Division of Critical Care Medicine, Sunnybrook Health Sciences Centre, University of Toronto, Toronto, Canada); Rajyabardhan Pattnaik (Ispat General Hospital, Rourkela, India); Jennifer L. Pigoga (Division of Emergency Medicine, University of South Africa, Cape Town, South Africa); Luigi Pisani (Department of Anesthesia and Intensive Care, Miulli Regional Hospital, Acquaviva delle Fonti, Italy; Doctors with Africa - CUAMM, Padova, Italy; Mahidol-Oxford Tropical Medicine Research Unit (MORU), Mahidol University, Bangkok, Thailand); Elisabeth D. Riviello (Division of Pulmonary, Critical Care, and Sleep Medicine, Beth Israel Deaconess Medical Center and Harvard Medical School, Boston, MA); Kristina E. Rudd (University of Pittsburgh School of Medicine, Pittsburgh, PA); Marcus Schultz (Mahidol University, Bangkok, Thailand; University of Oxford, Oxford, United Kingdom; Amsterdam University Medical Centers, Location 'AMC', Amsterdam, The Netherlands); Varun U. Shetty (University of Pittsburgh Medical 
Center, Pittsburgh, PA); Gentle S. Shrestha (Tribhuvan University Teaching Hospital, Kathmandu, Nepal); Chaisith Sivakorn (Mahidol University, Salaya, Thailand); Shaurya Taran (Interdepartmental Division of Critical Care Medicine, Sunnybrook Health Sciences Centre, University of Toronto, Toronto, Canada); T. Eoin West (Division of Pulmonary, Critical Care, and Sleep Medicine, University of Washington, Seattle, WA).

Group members of the subgroup "Tracheostomy, Discharge, and Rehabilitation Measures": Marcus Schultz (Mahidol University, Bangkok, Thailand; University of Oxford, Oxford, United Kingdom; Amsterdam University Medical Centers, Location "AMC", Amsterdam, The Netherlands). Hanan Yusuf (College of Health Sciences, Addis Ababa University, Addis Ababa, Ethiopia), Gentle S. Shrestha (Tribhuvan University Teaching Hospital, Kathmandu, Nepal), T. Eoin West (Division of Pulmonary, Critical Care, and Sleep Medicine, University of Washington, Seattle, WA), and Alfred Papali (Atrium Health, Charlotte, NC).

\section{REFERENCES}

1. Chiesa-Estomba CM et al., 2020. Systematic review of international guidelines for tracheostomy in COVID-19 patients. Oral Oncol 108: 104844.

2. Lamb CR et al., 2020. Use of tracheostomy during the COVID-19 pandemic: American college of chest physicians/American association for bronchology and interventional pulmonology/ association of interventional pulmonology program directors expert panel report. Chest 158: 1499-1514.

3. McGrath BA et al., 2020. Multidisciplinary guidance for safe tracheostomy care during the COVID-19 pandemic: the NHS National Patient Safety Improvement Programme (NatPatSIP). Anaesthesia 75: 1659-1670.

4. McGrath BA et al., 2020. Tracheostomy in the COVID-19 era: global and multidisciplinary guidance. Lancet Respir Med 8: 717-725.

5. Mecham JC, Thomas OJ, Pirgousis P, Janus JR, 2020. Utility of tracheostomy in patients with COVID-19 and other special considerations. Laryngoscope 130: 2546-2549.

6. Michetti CP et al., 2020. Performing tracheostomy during the covid-19 pandemic: guidance and recommendations from the critical care and acute care surgery committees of the American association for the surgery of Trauma. Trauma Surg Acute Care Open 5: e000482.

7. Schultz $P$ et al., 2020. French consensus regarding precautions during tracheostomy and post-tracheostomy care in the context of COVID-19 pandemic. Eur Ann Otorhinolaryngol Head Neck Dis 137: 167-169.

8. Smith D et al., 2020. Tracheostomy in the intensive care unit: guidelines during COVID-19 worldwide pandemic. Am J Otolaryngol 41: 102578

9. Sommer DD et al., 2020. Recommendations from the CSO-HNS taskforce on performance of tracheotomy during the COVID-19 pandemic. J Otolaryngol Head Neck Surg 49: 23.

10. Takhar A et al., 2020. Recommendation of a practical guideline for safe tracheostomy during the COVID-19 pandemic. Eur Arch Otorhinolaryngol 277: 2173-2184.

11. Kumar GP et al., 2020. Airway management and related procedures in critically ill COVID-19 patients: position statement of the Indian society of critical care medicine. Indian J Crit Care Med 24: 630-642.

12. Chiang SS et al., 2020. Controversies in tracheostomy for patients with COVID-19: the when, where, and how. Respir Care 65: 1767-1772.

13. Chao TN et al., 2020. Outcomes after tracheostomy in COVID-19 patients. Ann Surg 272: e181-e186.

14. Floyd E, Harris SS, Lim JW, Edelstein DR, Filangeri B, Bruni M, 2020. Early data from case series of tracheostomy in patients with SARS-CoV-2. Otolaryngol Head Neck Surg 163: 1150-1152.

15. Choi SY et al., 2020. Safe surgical tracheostomy during the COVID-19 pandemic: a protocol based on experiences with Middle East Respiratory Syndrome and COVID-19 outbreaks in South Korea. Oral Oncol 109: 104861.

16. Volo Tet al., 2020. Elective tracheostomy during COVID-19 outbreak: to whom, when, how? Early experience from Venice, Italy. Eur Arch
Otorhinolaryngol (Epub ahead of print, $2020 \mathrm{Jul} 12)$. Available at: https://doi.org/10.1007/s00405-020-06190-6.

17. Martin-Villares C, Perez Molina-Ramirez C, Bartolome-Benito M, Bernal-Sprekelsen M; COVID ORL ESP Collaborative Group, 2020. Outcome of 1890 tracheostomies for critical COVID-19 patients: a national cohort study in Spain. Eur Arch Otorhinolaryngol (Epub ahead of print, 2020 Aug 4). Available at: https://doi.org/10.1007/s00405-020-06220-3.

18. Thal $A G$ et al., 2020. Tracheotomy in a high-volume center during the COVID-19 pandemic: evaluating the surgeon's risk. Otolaryngol Head Neck Surg (Epub ahead of print, 2020 Sep 1). Available at: https://doi.org/10.1177/0194599820955174.

19. Menegozzo CAM et al., 2020. Standardization of elective tracheostomies at the Central Institute of the Hospital das Clinicas in Sao Paulo during the COVID-19 pandemic. Rev Col Bras Cir 47: e20202574.

20. Mishra P, Jedge $P$, Kaushik M, Artham P, Kumari S, 2020. Our experience of tracheostomy in COVID-19 patients. Indian $J$ Otolaryngol Head Neck Surg (Epub ahead of print, 2020 Aug 10). Available at: https://doi.org/10.1007/s12070-020-02036$z$.

21. Aodeng $S$ et al., 2020. Safety and efficacy of tracheotomy for critically ill patients with coronavirus disease 2019 (COVID-19) in Wuhan: a case series of 14 patients. Eur J Cardiothorac Surg 58: 745-751.

22. Yeung E, Hopkins $P$, Auzinger G, Fan K, 2020. Challenges of tracheostomy in COVID-19 patients in a tertiary centre in inner city London. Int J Oral Maxillofac Surg 49: 1385-1391.

23. Picetti $E$ et al., 2020. Safety of bedside surgical tracheostomy during COVID-19 pandemic: a retrospective observational study. PLoS One 15: e0240014.

24. Zuazua-Gonzalez A et al., 2020. Surgical tracheostomies in COVID19 patients: indications, technique, and results in a second-level Spanish hospital. OTO Open 4: 2473974X20957636.

25. Tran K, Cimon K, Severn M, Pessoa-Silva CL, Conly J, 2012. Aerosol generating procedures and risk of transmission of acute respiratory infections to healthcare workers: a systematic review. PLoS One 7: e35797.

26. Available at: https://www.cdc.gov/coronavirus/2019-ncov/hcp/ infection-control-recommendations.html. Accessed June 25, 2020.

27. Tay JK, Khoo ML, Loh WS, 2020. Surgical considerations for tracheostomy during the COVID-19 pandemic: lessons learned from the severe acute respiratory syndrome outbreak. JAMA Otolaryngol Head Neck Surg 146: 517-518.

28. Inglis R, Barros L, Checkley W, Cizmeci EA, Lelei-Mailu F, Pattnaik R, Papali A, Schultz MJ, Ferreira JC for the COVID-LMIC Task Force and the Mahidol-Oxford Research Unit (MORU), 2021. Pragmatic recommendations for safety while caring for hospitalized patients with COVID-19 in low- and middle-income countries. Am J Trop Med Hyg 104 (Suppl): 11-22.

29. Vargas $\mathrm{M}$ et al., 2015. Tracheostomy procedures in the intensive care unit: an international survey. Crit Care 19: 291.

30. Abe T et al., 2018. Epidemiology and patterns of tracheostomy practice in patients with acute respiratory distress syndrome in ICUs across 50 countries. Crit Care 22: 195.

31. Botti C et al., 2020. Comparison of percutaneous dilatational tracheotomy versus open surgical technique in severe COVID19: complication rates, relative risks and benefits. Auris Nasus Larynx (Epub ahead of print, 2020 Oct 28). Available at: https:// doi.org/10.1016/j.anl.2020.10.014.

32. Bassi $\mathrm{M}$ et al., 2020. Is surgical tracheostomy better than percutaneous tracheostomy in COVID-19-positive patients? Anesth Analg 131: 1000-1005.

33. Riestra-Ayora J, Yanes-Diaz J, Penuelas O, Molina-Quiros C, Sanz-Fernandez R, Martin-Sanz E, 2020. Safety and prognosis in percutaneous vs surgical tracheostomy in 27 patients with COVID-19. Otolaryngol Head Neck Surg 163: 462-464.

34. Susanto I, 2002. Comparing percutaneous tracheostomy with open surgical tracheostomy. BMJ 324: 3-4.

35. McGrath BA, Pelosi P, Schultz MJ, Brenner MJ, 2020. Preoperative apnea trial and considerations regarding timing of tracheostomy in anesthetic planning for patient with COVID-19 disease. J Clin Anesth 67: 110013. 
36. Available at: https://www.ecdc.europa.eu/sites/default/files/ documents/COVID-19-Discharge-criteria.pdf. Accessed May 26, 2020.

37. Available at: https://www.cdc.gov/coronavirus/2019-ncov/hcp/ disposition-hospitalized-patients.html. Accessed May 26, 2020.

38. Zhou B, She J, Wang Y, MaX, 2020. The duration of viral shedding of discharged patients with severe COVID-19. Clin Infect Dis 71: 2240-2242.

39. Lan $L$ et al., 2020. Positive RT-PCR test results in patients recovered from COVID-19. JAMA 323: 1502-1503.

40. Wolfel $R$ et al., 2020. Virological assessment of hospitalized patients with COVID-2019. Nature 581: 465-469.

41. La Scola B et al., 2020. Viral RNA load as determined by cell culture as a management tool for discharge of SARS-CoV-2 patients from infectious disease wards. Eur J Clin Microbiol Infect Dis 39: 1059-1061.

42. Yuan $\mathrm{J}$ et al., 2020. The correlation between viral clearance and biochemical outcomes of 94 COVID-19 infected discharged patients. Inflamm Res 69: 599-606.

43. National Health Commission of the People's Republic of China, 2020. Diagnosis and Treatment Protocol for COVID-19 Patients, 8th Addition. Available at: http://regional.chinadaily.com.cn/ pdf/DiagnosisandTreatmentProtocolforCOVID-19Patients (Tentative8thEdition).pdf. Accessed December 3, 2020.

44. Available at: https://mspp.gouv.ht/newsite/documentation.php? page $=1 \&$ param $1=$ valu1\&param2 $=$ value2. Accessed May 25, 2020.

45. Available at: https://www.who.int/publications-detail/clinicalmanagement-of-covid-19. Accessed May 28, 2020.

46. CDC, 2020. Discontinuation of Transmission-Based Precautions and Disposition of Patients with COVID-19 in Healthcare Settings (Interim Guidance). Available at: https://www.cdc.gov/ coronavirus/2019-ncov/hcp/disposition-hospitalized-patients.html. Accessed December 3, 2020.

47. Available at: https://www.ecdc.europa.eu/sites/default/files/ documents/COVID-19-Discharge-criteria.pdf. Accessed May 25, 2020.

48. European CDC, 2020. Guidance for Discharge and Ending of Isolation of People with COVID-19. Technical Report. Available at: https://www.ecdc.europa.eu/sites/default/files/documents/ Guidance-for-discharge-and-ending-of-isolation-of-people-withCOVID-19.pdf. Accessed December 3, 2020.

49. Lam PW et al., 2020. A virtual care program for outpatients diagnosed with COVID-19: a feasibility study. CMAJ Open 8: E407-E413.

50. The Indian Express, 2020. The Indian Express. Available at: https:// indianexpress.com/article/india/covid-19-test-pricesrates-india-6896237/. Accessed December 3, 2020.

51. Halpin SJ et al., 2020. Postdischarge symptoms and rehabilitation needs in survivors of COVID-19 infection: a cross-sectional evaluation. J Med Virol 93: 1013-1022.

52. Sheehy LM, 2020. Considerations for postacute rehabilitation for survivors of COVID-19. JMIR Public Health Surveill 6: e19462.

53. Stam HJ, Stucki G, Bickenbach J, 2020. COVID-19 and post intensive care syndrome: a Call for action. J Rehabil Med 52: jrm00044.

54. van Walraven C, Mamdani M, Fang J, Austin PC, 2004. Continuity of care and patient outcomes after hospital discharge. J Gen Intern Med 19: 624-631.

55. Smith JM et al., 2020. Home and community-Based physical therapist management of adults with post-intensive care syndrome. Phys Ther 100: 1062-1073.

56. World Health Organization, 2017. Rehabilitation 2030: A Call for Action. Available at: https://www.who.int/rehabilitation/rehab2030-call-for-action/en/. Accessed December 3, 2020.

57. Sigera PC et al., 2016. National profile of physical Therapists in critical care units of Sri Lanka: lower middle-income country. Phys Ther 96: 933-939.

58. Lyadov KV, Koneva ES, Polushkin VG, Sultanov EYu, Lukashin MA, 2020. Randomized controlled study on pulmonary rehabilitation in COVID-19 patients with pneumonia. Pul'monologiya 30: 569-576.
59. Liu K, Zhang W, Yang Y, Zhang J, Li Y, Chen Y, 2020. Respiratory rehabilitation in elderly patients with COVID-19: a randomized controlled study. Complement Ther Clin Pract 39: 101166.

60. Yang LL, Yang T, 2020. Pulmonary rehabilitation for patients with coronavirus disease 2019 (COVID-19). Chronic Dis Trans/ Med 6: 79-86.

61. Chinese Association of Rehabilitation, Respiratory Rehabilitation Committee of Chinese Association of Rehabilitation, Cardiopulmonary Rehabilitation Group of Chinese Society of Physical Rehabilitation, 2020. Recommendations for respiratory rehabilitation of coronavirus disease 2019 in adult. Zhonghua Jie $\mathrm{He} \mathrm{He} \mathrm{Hu}$ Xi Za Zhi 43: 308-314.

62. Zhao HM, Xie YX, Chen W, 2020.Recommendations for respiratory rehabilitation in adults with coronavirus disease 2019. Chin Med J 133: 1595-1602.

63. Spruit MA, Holland AE, Singh SJ, Tonia T, Wilson KC, Troosters T, 2020. COVID-19: interim guidance on rehabilitation in the hospital and post-hospital phase from a European respiratory society and American Thoracic society-coordinated international Task Force. Eur Respir J 56: 2002197.

64. Schofield-Robinson OJ, Lewis SR, Smith AF, McPeake J, Alderson $P, 2018$. Follow-up services for improving long-term outcomes in intensive care unit (ICU) survivors. Cochrane Database Syst Rev 11: CD012701.

65. Kamdar BB et al., 2020. Return to work after critical illness: a systematic review and meta-analysis. Thorax 75: 17-27.

66. Carter C, Snell D, 2016. Nursing the critically ill surgical patient in Zambia. Br J Nurs 25: 1123-1128.

\section{APPENDIX}

Development of recommendations and suggestions.

Selection of Task Force members. The selection of the group members was based on interest in specific aspects of COVID-19 and direct experience in LMICs. Alfred Papali and Marcus Schultz contacted potential team members through e-mail and in person early in the pandemic of COVID-19 and created 10 subgroups assigned to separate areas in COVID-19 management: "triage," "safety," "organization," "diagnostics," "acute respiratory failure," "acute kidney injury," "coagulopathy," "therapeutics," "shock," and "support after initial care." In total, there were 38 Task Force members representing five medical specialties or disciplines (emergency medicine, intensive care, infectious diseases, internal medicine, and critical care nursing) from five of six $\mathrm{WHO}$ geographic regions. The Task Force consisted of 16 full-time LMIC members, 16 full-time HIC members-all with direct LMIC experience - and six members with joint LMIC/HIC appointments.

TABLE A1

Quality of evidence

\begin{tabular}{lll}
\hline A & Randomized clinical trials & High \\
B & $\begin{array}{c}\text { Downgraded randomized clinical trial(s) } \\
\text { or upgraded observational studies }\end{array}$ & Moderate \\
C & $\begin{array}{l}\text { Observational studies } \\
\text { Downgraded observational studies or }\end{array}$ & Low \\
D & expert opinions &
\end{tabular}

Factors that could decrease the strength of evidence included a high likelihood of bias; Fistency of results, including problems with subgroup analyses; indirectness of evidence (other population, intervention, control, outcomes, and comparison); imprecision of findings; and likelihood of reporting bias. Factors that could increase the strength of evidence included large magnitude of effect (direct evidence, relative risk $>2$ with no plausible confounders); very large magnitude of effect with relative risk $>5$ and no threats to validity (by two levels); and dose-response gradient. ${ }^{6}$

The subgroup members paid extensive attention to several other factors as used before but now focusing on LMICs, that is, availability, feasibility, affordability and safety in LMICs. A strong recommendation was worded as "we recommend" and a weak recommendation as "we sugges"' (Appendix Table2). Recommendations could remain UG, when, in the opinion of the subgroup members, such recommendations were not conducive for the process described earlier. 
TABLE A2

Strong vs. weak recommendations *

What is Considered

High evidence

Certainty about the balance of benefits vs.

harms and burdens

Certainty in or similar values

Resource implications

Availability and feasibility in LMICs

Affordability for LMICs

Safety of the intervention in LMICs

Adapted from Ref. 67.

*In case of a strong recommendation, we use "we recommend ..."; in case of a weak recommendation, we use "we suggest ..."

Selection of subgroup members Alfred Papali, Marcus J. Schultz, Hanan Y. Ahmed, Gentle S. Shrestha, and T. Eoin West were assigned to this subgroup based on their specific expertise and interest in this area for patients with COVID-19.

Meetings. The subgroup worked via electronic-based communications to establish the procedures for the literature review, develop relevant questions, and drafting of tables for evidence analysis. Several electronic-based discussions among the subgroup leaders and members occurred.

The first step was to formulate a set of clearly defined questions regarding "support after initial care" for patients and healthcare workers caring for patients with COVID-19. An initial list of potential questions was reviewed for content and clarity by the subgroup members and leaders from the other subgroups, and questions were revised, adjusted, or eliminated after extensive discussion and according to group consensus. This process resulted in four distinct questions, two related to tracheostomy, one related to rehabilitation, and one related to post-hospitalization sequelae of severe COVID-19 disease.

Search techniques. In the next step, the "support after initial care" subgroup assigned one or two members to search the literature for evidence pertaining to each respective question. The literature search followed the same techniques as previously described. ${ }^{67}$ The literature search was performed in a minimum of one general database (i.e., MEDLINE and EMBASE) and the Cochrane Libraries, including articles pertaining to COVID-19, SARS, MERS, and other respiratory viruses. Specific efforts were made to emphasize literature based on LMICs, but in cases where no LMIC results were found, we appraised the literature from HICs. Furthermore, the subgroup members also searched for unpublished study results and included references suggested by group members, when relevant.

Grading of Recommendations. The subgroup members classified the quality of evidence as high, moderate, low, or very low and recommendations as strong or weak. The factors influencing this classification are presented in Appendix Table 1.

Reporting. The report was edited for style and form by Alfred Papali or Marcus Schultz, with final approval by the subgroup leaders and then by the entire "COVID-LMIC Task Force" in two rounds. A final document was submitted to the "American Journal of Tropical Medicine and Hygiene" for potential publication and made open access.

Conflicts of interest. No members of the "support after initial care" subgroup represented industry, and there was no industry input into recommendation development. No member of the "support after initial care" subgroup received honoraria for any role in the recommendation development process and none reported conflicts of interest. Open access fees for this manuscript, and all nine others in the series were supported by the Wellcome Trust of Great Britain.

\section{REFERENCE}

67. Dondorp AM, Dünser MW, Schultz MJ, eds., 2019. Sepsis Management in Resource-Limited Settings. Springer. 